\title{
Tolerancia de los estudiantes de pregrado ante los comportamientos desviados en el aula de clase: un estudio comparativo
}

Jorge Luis Avellaneda Suárez ${ }^{1}$

Recibido: 10 de enero de 2013

Aprobado: 15 de mayo de 2013

\begin{abstract}
Avellaneda, J. L. (2013). Tolerancia de los estudiantes de pregrado ante los comportamientos desviados en el aula de clase: un estudio comparativo. Activos, 20, 27-52
\end{abstract}

\section{Clasificación JEL K11}

\section{Resumen}

Los comportamientos desviados de los estudiantes a lo largo de su formación académica son más frecuentes de lo que se cree. De hecho, detrás del simple hecho de infringir una norma establecida por alguna institución educativa, hay una serie de construcciones sociales que se gestan alrededor de esta situación, y sobre esto es importante ahondar. En efecto, el enfoque del presente estudio es encontrar la postura y la tolerancia que tienen los estudiantes de dos Facultades diferentes frente al fraude académico, y tratar de encontrar las posibles causas de esa tolerancia ante las acciones desviadas. Además, se hará un énfasis especial en la Facultad de Administración, dadas la importancia y la relevancia que tienen los egresados de este programa académico en el desarrollo económico de un país.

1 Administrador de Empresas con Mención en Sociología, egresado de la Universidad del Rosario. Correo electrónico: avellaneda.jorge@ur.edu.co 


\title{
Palabras clave
}

Deshonestidad académica, ética, fraude, tolerancia al delito

Avellaneda, J. L. (2013). Undergraduate students' tolerance toward deviant behavior in the classroom: A comparative study. Activos, 20, 27-52

\begin{abstract}
Deviant behavior of students throughout their academic training is more common than believed. In fact, behind the simple act of breaking a rule established by any educational institution, there are a number of social constructions that are developing around this situation and it is important to delve into them. Indeed, the focus of this study is to find the position and tolerance that students from two different Faculties have toward academic fraud and try to find the possible causes for that tolerance towards deviant actions. In addition, there will be a special emphasis placed on the Faculty of Management, given the importance and relevance that graduates from this academic program have in the economic development of a country.
\end{abstract}

\section{Keywords}

Academic dishonesty, ethics, fraud, crime tolerance

Avellaneda, J. L. (2013).Tolérance des étudiants de premier cycle face aux comportements déviants dans la salle de classe : une étude comparative. Activos, 20, 27-52

\section{Jorge Luis Avellaneda Suárez}

\section{Résumé}

Les comportements déviants des élèves tout au long de leur formation académique sont plus fréquents que l'on le croit. En fait, derrière le simple fait de briser une règle édictée par n’importe quel établissement d’enseignement, 
il y a un certain nombre de constructions sociales qui est conçu autour de cette situation, et qui, surtout,est important d'approfondir. En effet, le but de cette étude est de trouver la posture et la tolérance qu'ont les étudiants des deux facultés différentes face à la fraude scolaire et essayer de trouver les causes possibles de cette tolérance aux actes déviants. En outre, on mettra l'accent particulièrement sur l'école de gestion, étant donné l'importance et la pertinence des diplômés de ce programme détudes dans le développement économique d’un pays

\section{Mots clés:}

malhonnêteté académique, éthique, fraude, tolérance pour ledélit

\section{Introducción}

En la actualidad, la necesidad de inclusión de posturas éticas en los negocios y la economía es un fenómeno que ha adquirido bastante relevancia dentro de la sociedad. Es una tendencia que se ha adherido a los mercados y bajo dicho concepto, la ética y los negocios buscan ir en armonía. Lipovetsky (2003) identificó cuatro factores que catalizaron dicha necesidad como un aspecto clave en el momento de desarrollar actividades comerciales; estos factores empezaron a reconfigurar la forma de concebir las transacciones entre los diferentes actores de los mercados.

El primer factor que logró identificar este sociólogo fue la aceleración en la toma de conciencia por parte de la población y las instituciones que regulan el medio ambiente frente a los desastres producidos por las industrias. La sucesión de irreversibles impactos en el medio ambiente empezaron a ser cada vez más frecuentes: derramamiento de petróleo crudo en varias costas, mal manejo de residuos tóxicos, aumento de la polución, mano de obra y poblaciones gravemente enfermas a causa de la radiación emitida en sus lugares de trabajo, entre muchas otras. Entonces, la población civil y las instituciones empezaron a entender la importancia de la ética dentro de los negocios y a demandarla. El segundo factor es un nuevo modelo económico 
del capitalismo inducido por políticas neo-liberales. Las transformaciones e innovaciones financieras que ha experimentado el sistema hacen más complejos los procesos de intercambio bursátil en los mercados internacionales. Estos procesos son cada vez más sofisticados, pero las medidas que regularizan las firmas son laxas por parte de las instituciones encargadas de realizar un control sobre las mismas. Lo anterior ha producido especulación en los precios de las acciones, transacciones ilícitas y corrupción; de esta forma, se hace necesaria la ética y una regularización que proteja los intereses de la sociedad. El tercer factor es la promoción del referente ético de la empresa a través del marketing, todo esto con el fin de abarcar más mercado. De esta forma, la ética funciona como un marketing de valores empresariales, estrategias comerciales y canales de comunicación, con el fin de conquistar nuevos mercados y ampliar en estos su participación. Además, el aspecto ético dentro de la empresa supone ser un nuevo elemento de valorización y posicionamiento ante sus competidores. Finalmente, el último factor es cómo los medios de comunicación han logrado denunciar y transmitir los actos de corrupción de las empresas, instituciones e individuos de la sociedad, quienes con sus acciones afectan los intereses de la sociedad en general. Se ha producido un cambio en concebir y difundir la noticia de forma más rápida y con una cobertura mucho más amplia. Por consiguiente, la población civil y la misma sociedad se enteran de esos casos particulares y demandan que la ética no sea un eslabón suelto dentro del mercado, sino que haga parte fundamental de las actividades económicas.

Por lo tanto, dentro de ese contexto, las empresas necesitan profesionales que cumplan con los requisitos propios del mercado y que logren satisfacer, paralelamente, los referentes éticos y el acatamiento de la ley. De esta forma, surge la necesidad de incluir la ética dentro de los negocios como un requisito fundamental para el desarrollo sostenible de la economía, y esto se reconoce como una tendencia de talante mundial. Ahora bien, actualmente en Colombia se ha venido presentando un esfuerzo por introducir la ética en el transcurrir de los negocios, que corresponde a la necesidad de crear un escenario que garantice un modelo de desarrollo económico transparente y sostenible. Se busca, entonces, propiciar un contexto ideológico, económico, cultural y político que propenda y exija que las transacciones 
comerciales no se encuentren en disonancia con el medio ambiente, las leyes y los valores propios de una sociedad.

Desde esas necesidades propias del mercado, es a los estudiantes de Administración a quienes les recaerá, en un futuro, la responsabilidad de cumplir con esas demandas del sistema económico y de la sociedad. Se espera de ellos que adquieran conocimientos técnicos, pero también formación moral y ética, cuyo resultado sea un profesional con excelentes aptitudes y competencias, pero al mismo tiempo, íntegro y moralmente aprobado por la sociedad. Sin embargo, la cuestión antagónica, contradictoria y muy preocupante es que los estudiantes tienen prácticas poco éticas a lo largo de su formación profesional dentro de la universidad. La copia o fraude, como infracción que va en contra de los lineamentos normativos de las instituciones educativas, se presenta con más frecuencia de lo que se cree entre los estudiantes. Además, alrededor de este fenómeno se entretejen una serie de construcciones sociales que son importantes de entender para tratar de minimizar esta problemática.

El presente estudio tratará de establecer algunas relaciones en cuanto a la tolerancia del fraude académico y su porqué, relacionando estadísticamente algunas variables. Se pretende replicar el estudio realizado por Magnus, Polterovich, Danilov y Savvateev (2011), llamado Tolerance of Cheating: An Analysis across Countries, incluyendo algunas preguntas adicionales para complementar el modelo. En la primera parte del presente estudio se realizó una revisión dela literatura sobre el tema del fraude académico; en la segunda parte se revisaron dos enfoques para ampliar el marco de referencia sobre este fenómeno, desde la economía y desde la sociología; en la tercera parte se plantea la metodología; en la cuarta se muestran los datos de la investigación, se exponen los resultados obtenidos y se presentan las conclusiones.

\section{Revisión de la literatura}

Con el propósito de comprender la importancia y complejidad de este fenómeno, se ha revisado la literatura relacionada con los comportamientos 
desviados dentro del aula de clase y los principales resultados de las investigaciones.

\subsection{La incidencia del fraude académico}

Aparentemente, las consecuencias del fraude académico se manifiestan cuando al estudiante se le asigna una nota más alta de la que merece, entonces los estudiantes que sí estudian para sus evaluaciones se desmotivan y perciben una posición injusta; además, los métodos de evaluación no cumplen su propósito, porque no se puede determinar si el estudiante aprueba verdaderamente los requerimientos de cada curso. A pesar de esto, existen otras incidencias en las pautas de comportamiento que no son tan evidentes en los estudiantes que toleran la copia y/o incurren en ella, y que se ven reflejadas a largo plazo y en escenarios distintos al universitario.

A partir de los resultados obtenidos por Sims (1993), en los cuales se refleja una correlación directa entre el fraude del pasado y el fraude del futuro, los autores Smyth, Davis \& Kroncke (2009) desarrollaron una investigación que concluye que si una persona tiene comportamientos no éticos en las aulas de clase, existe una correlación directa con que en el trabajo también siga esas pautas de comportamiento no éticas y alejadas de la norma. Estos autores, en sus resultados, reflejaron que la copia sí tiene una repercusión en el desempeño laboral. Según lo anterior, la incidencia del fraude académico va más allá de una simple nota inmerecida, pues esos comportamientos desviados se replican y extrapolan en otras áreas de la vida. Ahora bien, la muestra del estudio se realizó con base en estudiantes de Administración de Empresas, lo cual implica que a las organizaciones a las cuales ingresarán los egresados en un futuro, no están exentas de experimentar posibles actos de corrupción o comportamientos desviados, si lo estudiantes tuvieron comportamientos poco éticos en el aula de clase. 


\subsection{Culpa compartida}

Chisholm (1992) afirma que la deshonestidad no puede ser erradicada completamente, y esa deshonestidad inevitable la denomina "deshonestidad estructural". A pesar de que existe esa deshonestidad estructural, este autor afirma que los profesores deben dirigir todos sus esfuerzos a tratar de reducir al máximo los comportamientos desviados que irrumpen con la normatividad moral establecida. Acepta que una gran responsabilidad de que la deshonestidad se fomente recae sobre el profesor, porque puede promover la copia si utiliza los mismos exámenes vez tras vez, si crea una fama de facilismo en su materia o si es muy laxo, entre otras. Estas apreciaciones están relacionadas con los planteamientos desde un enfoque económico sobre el crimen, los cuales serán ampliados más adelante.

Murdock, Beauchamp \& Hinton (2008) trataron de establecer cómo el ambiente en el aula de clase influye en que los estudiantes incurran en el fraude académico. Los estudiantes construyen argumentos para autojustificarse al momento de copiarse, y en la mayoría de los casos esos argumentos recaen sobre el profesor. Concluyen que los estudiantes aceptan incurrir en el fraude académico cuando el profesor desarrolla el curso con una pedagogía inadecuada, es decir, cuando el profesor no tiene un manejo apropiado de la clase.

Así que, aunque el estudiante asume la responsabilidad de sus actos y las decisiones que toma, no se puede obviar la importancia del profesor en este fenómeno del fraude académico. El rol que el profesor desempeña es fundamental para comprender estas acciones que se desvían de la norma.

\subsection{La educación como una competencia}

Bouville (2010) plantea una discusión atípica sobre las razones que permiten concluir que la copia es un acto moralmente incorrecto y centra su discusión en dos premisas que generalmente son utilizadas para justificar que la copia académica está mal: las desventajas que se producen cuando un estudiante incurre en la copia y que la copia es un obstáculo para el aprendizaje. 
Entonces, este autor elabora algunos argumentos para controvertir esos planteamientos, los cuales son comúnmente aceptados.

En primer lugar, cuando se trata de una posición injusta, es decir, cuando un estudiante utiliza otros elementos que crean algún tipo de desigualdad en las oportunidades al momento de responder algún examen, dicho estudiante ha creado una desventaja a su favor en comparación con los otros estudiantes. Aunque ese escenario se presenta frecuentemente en los exámenes, no se logra tener conciencia sobre la desventaja y desigualdad entre los estudiantes. Ningún estudiante va a tener el mismo conocimiento, ni la misma experiencia, ni el mismo capital cultural que los demás, y viceversa, para poder decir que están en "igualdad" de condiciones cuando responden un examen. Entonces, este autor encuentra que el argumento para justificar que la copia es incorrecta, basado en la desigualdad de oportunidades, es una argucia, ya que la desigualdad de oportunidades entre estudiantes siempre existirá.

En segundo lugar, cuestiona las drásticas sanciones por plagiar y/o copiarse por parte de las instituciones educativas, ya que han creado sanciones tan duras, que lo que se está logrando es un daño y perjuicio grave contra el estudiante, pues ese tipo de sanciones sí impide que el estudiante siga dentro de los procesos de formación y aprendizaje. En la mayoría de los casos se expulsa al estudiante de la institución, pero esto, ¿cómo contribuye dentro de sus procesos de aprendizaje?

Sin embargo, detrás de ese polémico debate, se deja al descubierto una situación álgida en cuanto al modelo educativo que se desarrolla actualmente, pues se ha convertido en una carrera de todos contra todos por tratar de conseguir una buena calificación, sin importar si verdaderamente se está logrando el objetivo de la asignatura o del programa académico. Se convierte en una especie de fetiche el obtener la mejor nota sin importar nada; se ha convertido en un modelo de competencia directa entre los estudiantes; en otras palabras, quien tiene la mejor nota, se convierte en el mejor estudiante. Por lo tanto, la discusión que plantea este autor sobre controvertir los fundamentos en los cuales descansa la norma y la sanción amplían mucho más el debate y el modelo de educación. 


\subsection{Delimitando el fraude académico}

Según Colnerud y Rosander (2009), este comportamiento desviado puede tener tres categorías, teniendo en cuenta el grado de conciencia y la intención al realizarlo por parte del estudiante:

- Fraude consciente: se caracteriza porque el estudiante trata de enviar o envía un mensaje tergiversado, intencionalmente, sobre sus acciones. Es decir, da a entender que él hizo algo que realmente no hizo. El individuo toma una decisión racional de infringir una norma establecida.

- Auto-engaño: se caracteriza por una auto-manipulación, y el estudiante considera que ha realizado un trabajo que él no hizo. Lo curioso de este caso es que el estudiante no considera que ha cometido una infracción, pero sí lo ha hecho.

- Fraude en ignorancia: se caracteriza por la ignorancia del estudiante frente a la norma y las pautas de comportamiento que están socialmente aceptadas dentro de un contexto específico.

Además, dentro de los diferentes comportamientos de los estudiantes, existen diferentes acciones que se alejan de los parámetros normativos y sociales de las instituciones, como por ejemplo firmar la asistencia a clase en nombre de otro estudiante que no está, o copiar citas textuales de otros autores sin denotar las referencias indicadas. Entonces, con el fin de delimitar el tema para el presente estudio, el fraude se concebirá como un acto consciente y en los momentos específicos de presentar un examen escrito.

\section{El crimen desde dos perspectivas}

Según Morin (1999), la realidad es bastante compleja y cuando se realiza un acercamiento a algún fenómeno, se tiende a reducir y simplificar dicho fenómeno. Sin embargo, la realidad no puede ser desfragmentada de esa forma y por esto, se hace casi indispensable que los estudios tengan una perspectiva interdisciplinaria, con el fin de ampliar el marco de referencia y plantear posibles acciones alternativas que incidan en la problemática. En este caso, el crimen se aborda con dos enfoques, desde la Sociología y 
desde la Economía, con el propósito de complementar diferentes posturas y construir posibles soluciones.

\subsection{El crimen desde la Economía}

Según Becker (1968), el crimen es un acto racional, porque el delincuente potencial desea maximizar sus beneficios y entonces pondera los costos y los beneficios de cometer un delito. Los costos son entendidos como el posible riesgo de ser capturado y castigado; de esta forma se convierte en un caso del comportamiento racional bajo incertidumbre (teoría de juegos). Así, Becker involucra el concepto de utilidad esperada, y sobre éste el individuo decide si procede o no a cometer el delito. En términos prácticos y aplicándolo a nuestro tema de interés, si el estudiante encuentra que la probabilidad de ser sorprendido copiándose o cometiendo un fraude académico es baja, el castigo no es tan drástico ni ejemplar y/o los incentivos son altos cuando obtiene un excelente resultado sin necesidad de algún esfuerzo; entonces, procederá a cometer esa infracción. De esta forma, el fenómeno de la copia o fraude no depende únicamente del estudiante, sino también del profesor, de las instituciones y sus políticas disciplinarias, y aún de los compañeros de clase. El crimen, en este caso, se convierte en una solución más atractiva para conseguir buenos resultados académicos, ya que con otros mecanismos y acciones se consigue la nota (el incentivo en este caso) sin necesidad de largas horas de estudio ni un esfuerzo considerable por parte del estudiante. La incertidumbre de la pena depende de algunos factores como la postura del profesor ante la copia, las políticas disciplinarias de la institución educativa en casos de fraude y la tolerancia de los compañeros de clase.

\subsection{El crimen desde la Sociología - Teoría funcionalista}

Giddens (2006) ve la importancia de estudiar el crimen y la desviación social porque son las instituciones sociales las que definen las normas y valores de una sociedad, y por ende, le compete a la Sociología hacer un acercamiento minucioso al tema. Este autor hace una marcada diferencia entre crimen y desviación social, ya que el crimen es entendido como la acción que vulnera 
una ley, mientras que la desviación social va mucho más allá de violar una ley, pues es una inconformidad hacia las normas establecidas, y alrededor de esa acción que vulnera la ley se gestan ciertas construcciones sociales que se hacen habituales. La desviación social depende directamente del contexto social, de las normas, las leyes, de quiénes hacen las leyes, de las penas o castigos por infringir las normas, en fin; depende de varios elementos contextuales. Dentro de las sociedades existen normas formales e informales. Las primeras se establecen como ley y su incumplimiento acarrea una sanción punitiva; las segundas se establecen a partir de las costumbres propias de los individuos y su cultura, y las sanciones son simbólicas o sociales. Entender esta diferencia es relevante, pues esos códigos culturales que se gestan alrededor del control social son los que permiten entender la configuración de los valores propios de una sociedad.

Para este caso, la copia o fraude académico está enmarcado dentro de un reglamento y se convierte en una ley de obligatorio cumplimiento, pues el individuo que la infrinja es castigado con una sanción punitiva y no simbólica. Además, Giddens (2009) recopiló las teorías de dos de los autores más influyentes dentro de la Sociología, Emile Durkheim y Robert Merton, y condensó sus mayores aportes, que explican el crimen dentro de la sociedad. Para la teoría funcionalista, el crimen y la desviación social se dan como consecuencia de las tensiones estructurales y la ausencia de una regulación moral dentro de la sociedad. Es decir, cuando los deseos de los individuos y/o grupos sociales no corresponden con las recompensas disponibles, esa coyuntura entre el deseo y la imposibilidad de lograrlo es un escenario propicio para que se dé el crimen. En palabras más sencillas, cuando la estructura de la sociedad propone una serie de metas u objetivos, pero su misma estructura y funcionamiento hacen que solo pocos individuos logren alcanzar esas expectativas o metas, los individuos buscan conseguir tan anheladas metas cueste lo que cueste. Aplicando estos planteamientos en nuestro caso de estudio, se presenta el delito cuando la materia es demasiado exigente (difícil académicamente) y la expectativa del estudiante es conseguir una buena calificación, entonces encontramos una tensión entre la estructura y las aspiraciones del estudiante. 


\section{Metodología}

El presente estudio utilizó como punto de referencia el trabajo desarrollado por Magnus, Polterovich, Danilov y Savvateev (2011), en el cual se realizaron unas encuestas en diferentes países (Rusia, Estados Unidos, Israel y Países Bajos) a estudiantes con diferentes niveles de educación (secundaria, pregrado y posgrado), y se les indicó que evaluaran entre una escala de (-2) a (2) tres diferentes actores en el siguiente escenario: el estudiante (A) se dirige a la facultad para reportar a un estudiante (B) que se copió del estudiante (C). El estudiante (B) se copió con el consentimiento del estudiante (C). Entonces, para nuestro caso se utilizó el mismo modelo y se incluyeron cinco preguntas adicionales con el fin de complementarlo.

$$
y_{i}=\left\{\begin{array}{l}
-2 s i y^{{ }_{i}} \leq \tau_{0} \\
-1 \text { si } \tau_{0}<y^{*}{ }_{i} \leq \tau_{1} \\
0 s i \tau_{1}<y^{*}{ }_{i} \leq \tau_{2} \\
2 s i y^{*_{i}}>\tau_{3}
\end{array}\right\}
$$

En la muestra que se utilizó se empleó una variable dummy, $x_{1}$, la cual toma el valor de 1 si el estudiante pertenece a la Facultad de Administración y 0 para los estudiantes pertenecientes a la Facultad de Ciencias Humanas. De igual forma, con el género de cada encuestado se utilizó otra variable dummy, $x_{2}$, la cual se le asignó el valor de 1 para los hombres y 0 para las mujeres.

Para la primera pregunta, que busca encontrar algún tipo de justificación ante los comportamientos desviados, se asignó una variable dummy $x_{3}$ que tomaba el valor de 1 para cualquier tipo de justificación y 0 para la opción que indicaba que la copia o fraude académico es justificable. Para la segunda pregunta, en la cual se pretendía conocer si alguna vez ha visto a algún compañero de clase copiarse o incurrir en fraude académico, se asignó una variable dummy $x 4$ que tomaba el valor de 1 para la respuesta afirmativa y 0 para respuesta negativa. La tercera pregunta iba relacionada con la anterior, pues de ser afirmativa la respuesta, se preguntaba si el estudiante fue sorprendido por el profesor. Esta pregunta busca ver qué 
tan fuerte es el control por parte de los profesores. La cuarta pregunta buscaba encontrar si los estudiantes habían leído la norma que regula el tema de la copia o fraude. Entonces, se le asignó una variable dummy $x 5$ que tomaba el valor de 1 si había leído el reglamento y 0 si no lo había leído. En la pregunta cinco, se buscaba entender la percepción que tienen los estudiantes frente a considerar la copia como un problema, entonces se le asignó una variable dummy $x 6$ que daba el valor de 1 a la consideración de que la copia es un problema serio y 0 para las consideraciones de que es un problema trivial y que no es un problema. Además, con la variable de la edad se realizó una interacción con ella misma elevándola al cuadrado, con el fin de explicar mejor el comportamiento de la percepción de los estudiantes a lo largo del tiempo.

De este modo, y con las variables mencionadas, se corrió un modelo de regresión probit con el fin de entender las relaciones estadísticas más significativas, y cuáles eran las variables que más influían en la tolerancia que tienen los estudiantes ante los comportamientos desviados.

\section{Datos}

Con el fin de llevar a cabo el presente estudio sobre la tolerancia que tienen los estudiantes frente a los comportamientos desviados dentro del aula de clase, en el segundo semestre del año 2012 se realizaron 153 encuestas a estudiantes de la Facultad de Administración de la Universidad del Rosario y 83 encuestas a estudiantes de la Facultad de Ciencias Humanas de la misma Universidad, para un total de 236 encuestados. Es decir, el $65 \%$ fueron estudiantes de la Facultad de Administración y el 35 \% fueron estudiantes de la Facultad de Ciencias Humanas. Dentro de la muestra, el 47 \% eran hombres y el $53 \%$ eran mujeres, los cuales se encontraban en un rango entre los 16 y los 36 años de edad. Se encuestó a estudiantes de todos los semestres con el fin de entender la tolerancia de estos frente al fraude académico a lo largo de su formación académica, y cómo ésta varía a medida que van avanzando en los semestres. 


\subsection{Justificación de la copia}

La primera pregunta se diseñó con el fin de observar si el estudiante encontraba algún caso en el que la copia fuera, de alguna manera, justificable. Es decir, si el delito tiene alguna razón válida para que el individuo prefiera infringir la norma y tenga los incentivos suficientes que superen el posible castigo. Esta pregunta implica no solo que el fraude es un mero impulso, sino que permite ver que estos tipos de comportamientos son racionales. El $38 \%$ de la muestra en total encuentra una razón válida para infringir la norma sin importar el castigo que podría recibir si es sorprendido. Para la Facultad de Administración, el 44 \% de los estudiantes encuentra algún tipo de justificación para infringir la norma.

Tabla 1

Justificación de la copia o fraude académico

\begin{tabular}{|c|c|c|c|c|c|}
\hline \multirow{2}{*}{$\begin{array}{l}\text { ¿En cuál de las siguientes } \\
\text { situaciones considera que la } \\
\text { copia se justifica? } \\
\begin{array}{l}\text { Cuando hay presión por parte } \\
\text { de los padres }\end{array}\end{array}$} & \multicolumn{2}{|c|}{$\begin{array}{c}\text { Estudiantes } \\
\text { Administración }\end{array}$} & \multicolumn{2}{|c|}{$\begin{array}{c}\text { Estudiantes } \\
\text { Ciencias } \\
\text { Humanas }\end{array}$} & \multirow{2}{*}{$\begin{array}{r}\text { Total } \\
8\end{array}$} \\
\hline & 6 & $4 \%$ & 2 & $2 \%$ & \\
\hline Otra & 7 & $5 \%$ & 1 & $1 \%$ & 8 \\
\hline Cuando todo el curso lo hace & 13 & $8 \%$ & 2 & $2 \%$ & 15 \\
\hline $\begin{array}{l}\text { Cuando es para ayudar a un } \\
\text { amigo }\end{array}$ & 9 & $6 \%$ & 6 & $7 \%$ & 15 \\
\hline $\begin{array}{l}\text { Cuando la clase no es impor- } \\
\text { tante para su formación }\end{array}$ & 9 & $6 \%$ & 8 & $10 \%$ & 17 \\
\hline $\begin{array}{l}\text { Cuando la carga académica es } \\
\text { muy exigente }\end{array}$ & 23 & $15 \%$ & 4 & $5 \%$ & 27 \\
\hline $\begin{array}{l}\text { La copia o fraude académico } \\
\text { jamás es justificable }\end{array}$ & 86 & $56 \%$ & 60 & $72 \%$ & 146 \\
\hline
\end{tabular}

Fuente: Autor.

\subsection{Evidencias empíricas sobre el fraude}

La segunda pregunta se planteó tratando de encontrar si efectivamente algunos alumnos han incurrido en el fraude académico. Resulta más 
acertado preguntar si el estudiante ha visto a algún compañero copiarse, en vez de preguntar directamente al estudiante si se ha copiado. De esta forma, se buscaba ver si efectivamente la copia es un fenómeno relevante y con frecuencia dentro de la formación académica en pregrado. De 236 encuestados, 166 reportaron que vieron alguna vez a algún compañero incurrir en esta falta, es decir el 70,33 \% de la muestra. En la Facultad de Administración el porcentaje es de 75,81 \% y en la Facultad de Ciencias Humanas el porcentaje es de 60,24\%. Este resultado es un porcentaje bastante considerable, el cual refleja la relevancia de ahondar más en esta problemática y tratar de plantear posibles alternativas que se materialicen en acciones concretas para disminuir dicho fenómeno.

Tabla 2

Evidencias empíricas del fraude

\begin{tabular}{lcccccc}
\hline $\begin{array}{l}\text { En el actual semestre, ¿ha visto } \\
\text { a algún compañero incurrir en } \\
\text { fraude académico? }\end{array}$ & $\begin{array}{c}\text { Estudiantes } \\
\text { Administración }\end{array}$ & $\begin{array}{c}\text { Estudiantes } \\
\text { Ciencias } \\
\text { Humanas }\end{array}$ & Total \\
\hline Sí & 116 & $75,8 \%$ & 50 & $60,2 \%$ & 166 & $70 \%$ \\
No & 37 & $24,1 \%$ & 33 & $39,7 \%$ & 70 & $30 \%$ \\
\hline
\end{tabular}

Fuente: Autor.

\subsection{Los incentivos de infringir la norma}

La siguiente pregunta buscaba encontrar si los mecanismos de control por parte de los profesores son altos, ya que a menor control, mayor incentivo para infringir la norma. De esta manera, la pregunta estaba relacionada directamente con la anterior, pues de haber visto a algún compañero incurrir en el fraude, se pretendía indagar si éste fue descubierto o no. De los 166 estudiantes que fueron vistos por sus compañeros, únicamente 24 fueron sorprendidos por el profesor. Lo anterior nos da un referente importante para interpretar que los controles ante el fraude son fácilmente burlados, es decir, que la probabilidad de ser sorprendidos es muy baja, y el incentivo para infringir la norma aumenta. Para la Facultad de Administración, únicamente fueron sorprendidos por el profesor 17 estudiantes, es decir, 
el 14,66 \% de los estudiantes que fueron vistos por sus compañeros infringiendo la norma.

Tabla 3

Incentivos para infringir la norma

\begin{tabular}{lcrlrrr}
\hline $\begin{array}{l}\text { ¿El compañero } \\
\text { que se copió fue } \\
\text { descubierto? }\end{array}$ & \multicolumn{2}{c}{$\begin{array}{c}\text { Estudiantes } \\
\text { Administración }\end{array}$} & \multicolumn{2}{c}{$\begin{array}{l}\text { Estudiantes } \\
\text { Ciencias } \\
\text { Humanas }\end{array}$} & \multicolumn{2}{l}{ Total } \\
\hline Sí & 17 & $14,66 \%$ & 7 & $14 \%$ & 24 & $14 \%$ \\
No & 99 & $85,34 \%$ & 43 & $86 \%$ & 142 & $86 \%$ \\
\hline
\end{tabular}

Fuente: Autor.

\subsection{Conocimiento de la norma}

La interiorización de la norma para que el individuo la cumpla debe partir del conocimiento de la misma. Si el individuo no conoce la norma que regula algún tipo de comportamiento, es más difícil lograr que sus pautas de comportamiento se modifiquen a través de dicha norma. En este caso, se pretendía conocer cuántos estudiantes han leído la norma que regula el tema de la copia. Del total de los encuestados, el 25,8 \% han leído la norma que regula el tema de la copia y el fraude académico. Este porcentaje es bastante preocupante, pues se exige que se cumpla una norma, pero los estudiantes no tienen un conocimiento preciso sobre ella. Para la Facultad de Administración, el 71,90 \% de la muestra no ha leído la norma y el porcentaje aumenta para la Facultad de Ciencias Humanas, pues el 78,31 \% no ha leído la norma. En estos casos, es muy importante que el estudiante conozca en detalle la norma, ya que si no la conoce, no puede interiorizarla.

Tabla 4

Conocimiento de la norma

\begin{tabular}{lcrrrrr}
\hline $\begin{array}{l}\text { ¿Ha leído el } \\
\text { reglamento sobre el } \\
\text { fraude académico? }\end{array}$ & \multicolumn{2}{c}{$\begin{array}{c}\text { Estudiantes } \\
\text { Administración }\end{array}$} & \multicolumn{2}{l}{$\begin{array}{l}\text { Estudiantes } \\
\text { Ciencias } \\
\text { Humanas }\end{array}$} & \multicolumn{2}{c}{ Total } \\
\hline Sí & 43 & $28,10 \%$ & 18 & $21,69 \%$ & 61 & $25,85 \%$ \\
No & 110 & $71,90 \%$ & 65 & $78,31 \%$ & 175 & $74,15 \%$ \\
\hline
\end{tabular}

Fuente: Autor. 


\section{5 ¿La copia es un problema?}

La siguiente pregunta estaba enfocada hacia la percepción que tienen los estudiantes frente al fenómeno de la copia. Aunque la copia o fraude académico está dentro de la normatividad de la institución educativa como un acto moralmente incorrecto y con sanciones formales, la percepción de los estudiantes puede variar. De hecho, en este caso, el 45,34 \% tiene la percepción de que la copia no es un problema serio.

Tabla 5

Percepción de la copia

\begin{tabular}{lcrrrrr}
\hline Facultad & \multicolumn{2}{c}{$\begin{array}{c}\text { No es un } \\
\text { problema }\end{array}$} & \multicolumn{2}{c}{$\begin{array}{c}\text { Es un problema } \\
\text { trivial }\end{array}$} & $\begin{array}{c}\text { Es un problema } \\
\text { serio }\end{array}$ \\
\hline Administración & 7 & $58,33 \%$ & 63 & $66,32 \%$ & 83 & $64,34 \%$ \\
Ciencias Humanas & 5 & $41,67 \%$ & 32 & $33,68 \%$ & 46 & $35,66 \%$ \\
Total & \multicolumn{2}{c}{12} & \multicolumn{2}{c}{95} & \multicolumn{2}{c}{129} \\
\hline
\end{tabular}

Fuente: Autor.

\subsection{La tolerancia frente al comportamiento desviado}

La última pregunta, la cual fue extraída del estudio Tolerance of Cheating: An Analysis across Countries, mencionado anteriormente, pretende establecer el grado de tolerancia de los estudiantes ante comportamientos desviados. La información que se muestra a continuación es el promedio de la percepción de cada estudiante por facultad, hacia los tres estudiantes mencionados (A, B y C).

Se puede apreciar en las gráficas cómo son las tendencias y las diferencias en las percepciones de los estudiantes de diferentes facultades (en los anexos se encuentran las gráficas). En el primer caso del estudiante $A$ (el que reporta la copia) no se puede distinguir con precisión una tendencia, pero lo que sí se logra dilucidar es que los estudiantes de ambas Facultades no tienen una percepción positiva y son indiferentes sobre ese comportamiento (ver Gráfica 1 en Anexos). Los estudiantes de la Facultad de Administración 
reportan más veces como negativo ese comportamiento que en la Facultad de Ciencias Humanas.

En el segundo caso del estudiante B (el que se copia), claramente se evidencia una percepción negativa por parte de los estudiantes de ambas Facultades y lo interesante del ejercicio es que a medida que los estudiantes avanzan en semestres académicos, su tolerancia sobre este tipo de comportamientos disminuye. Una diferencia importante es que los estudiantes de la Facultad de Ciencias Humanas califican más negativamente al estudiante que infringe la norma, mientras que el estudiante de la Facultad de Administración, aunque lo califica como negativo, es más laxo y no lo considera tan negativo. Estos resultados son relevantes, pues nos permiten entender que a medida que aumenta la permanencia de los estudiantes dentro de una institución que trata de inculcar y trasmitir ciertas pautas de comportamiento, y en últimas, los valores que van formando la moral de los individuos, los estudiantes efectivamente se van apropiando de los valores y comportamientos que la institución transmite (ver Gráfica 2 en Anexos).

En el último caso, el estudiante $C$ (el que se deja copiar) tiene un resultado bastante similar en cuanto a la percepción de ambas Facultades, pues en promedio, ninguno lo evaluó como positivo. Obviamente algunos casos aislados, en total 16 personas, los calificaron como positivo; mientras que 113 personas de toda la muestra lo calificaron como indiferente. Ante este último comportamiento, en el que un individuo es cómplice con el delito, en la mayoría de los casos los estudiantes presenta una actitud de indiferencia (ver Gráfica 3 en Anexos).

\section{Resultado obtenidos}

A través del desarrollo de una regresión probit, se trató de establecer si existía alguna relación estadísticamente significativa que permitiera dilucidar cuáles variables influenciaban en el comportamiento tolerante ante la copia o fraude académico. A partir de observaciones empíricas y lecturas previas sobre el tema, se tomaron como referencia algunas variables que 
probablemente influirían en la postura de los estudiantes frente a la copia o fraude académico.

Se incluyeron variables como la Facultad a la que pertenecían los estudiantes, el género, la edad, la edad al cuadrado, si el estudiante ha visto a algún compañero incurrir en la falta, si el estudiante ha leído el código estudiantil que regula el tema del fraude académico, si el fraude es un problema y si encuentra alguna justificación para infringir la norma. De esta forma, se construyó un modelo probit y se demostró, estadísticamente, que las variables que influían en la tolerancia ante el fraude académico son la percepción que tiene cada individuo frente a la copia y la justificación que cada individuo tiene de ésta (ver Tabla 6). Es decir, cuando un estudiante tiene la percepción de que la copia no es un problema y encuentra algún tipo de justificación para realizar este tipo de acciones que infringen abiertamente la norma, hay una correlación con la tolerancia ante el fraude académico.

Lo interesante de los resultados obtenidos es que algunas de las variables que caracterizan propiamente al individuo (p. e. el género, el programa académico, el semestre) no tuvieron mucha relevancia en el estudio. Por ejemplo, se trató de hacer una comparación entre diferentes programas de pregrado y tratar de establecer si por la formación y enfoque propio de cada programa habría alguna incidencia en cuanto a la tolerancia a la copia o fraude académico; sin embargo, no la hubo. De igual forma, con el género y la edad se presentó la misma situación. Entonces, encontramos que es a partir de las concepciones propias del individuo y sus marcos de referencia que éste tiene más tolerancia o no frente al quebrantamiento de esta norma.

En cuanto a la edad de los individuos, se espera que a mayor edad haya una menor tolerancia a la copia, dado que el individuo adquiere un mayor grado de madurez conforme avanza el tiempo. Pero esto no es consistente con los resultados obtenidos. En el estudiante B, que es el que se copia, la edad tiene un efecto positivo en cuanto a la tolerancia a la copia, y el coeficiente obtenido es estadísticamente significativo. Si bien esto parece ir en contravía de la intuición, al analizar más en detalle el modelo, es posible 
concluir que el efecto marginal de la edad sobre la tolerancia a la copia, a pesar de ser positivo, es también marginalmente decreciente. Con base en esto, llegará un punto en la edad del individuo en que empiece a considerar la copia como un problema y deje de ser tolerante frente a ella, lo cual refleja que la madurez del individuo es un factor importante en cuanto al fraude.

\section{Conclusiones}

El presente trabajo expone un modelo empírico aplicado en estudiantes de pregrado de dos facultades diferentes, en el cual se ha demostrado que la tolerancia al fraude académico aumenta cuando el individuo tiene la percepción de que el fraude no es un problema serio y cuando construye algún tipo de justificación para realizar este tipo de acciones que infringen abiertamente la norma.

En el desarrollo del presente estudio, se obtuvo que un $38 \%$ del total de los estudiantes encuestados encontraba alguna razón válida para infringir la norma y copiarse de otro estudiante en busca de un fin: una buena nota. Este resultado empieza a dar visos de un problema en la educación, pues el fin último y per se de la educación se convierte en una buena calificación. Además, un resultado que debería ser un referente importante para nuevos debates son las evidencias empíricas sobre el fraude, pues el 75,81 \% de los estudiantes encuestados de la Facultad de Administración y el 60,24 \% de los estudiantes encuestados de la Facultad de Ciencias Humanas vieron a algún compañero de pregrado incurrir en la copia o fraude académico.

Estos resultados tan altos no pueden ser ignorados porque están alertando y dando algunas señales sobre los comportamientos de los estudiantes de pregrado. Confirman, además, la alta frecuencia de este fenómeno en las aulas de clase. Se puede afirmar, paralelamente, que existen altos incentivos en infringir la norma, ya que del total de las personas que fueron vistas por sus compañeros infringiendo la norma, únicamente el 14,45\% fueron sorprendidas por el profesor. Los estudiantes, entonces, tendrán altos incentivos en transgredir los reglamentos de la institución educativa 
Tabla 6

Resultados modelo probit

\begin{tabular}{|c|c|c|c|}
\hline & (1) & (2) & (3) \\
\hline VARIABLES & A & B & $\mathrm{C}$ \\
\hline \multirow[t]{2}{*}{ Administración } & $-0,012$ & $-0,062$ & $-0,047$ \\
\hline & 0,139 & 0,154 & 0,141 \\
\hline \multirow[t]{2}{*}{ Hombre } & $-0,011$ & $-0,003$ & $-0,032$ \\
\hline & 0,141 & 0,147 & 0,147 \\
\hline \multirow[t]{2}{*}{ Pregunta 2} & $-0,178$ & 0,162 & 0,219 \\
\hline & 0,155 & 0,171 & 0,156 \\
\hline \multirow[t]{2}{*}{ Pregunta 4} & 0,188 & $-0,117$ & $-0,157$ \\
\hline & 0,153 & 0,173 & 0,180 \\
\hline \multirow[t]{2}{*}{ Copia Problema } & $1,016^{* * *}$ & $-1,174^{\star * *}$ & $-1,471^{* * *}$ \\
\hline & 0,372 & 0,279 & 0,372 \\
\hline \multirow[t]{2}{*}{ Copia Justificable } & $-0,293^{*}$ & $0,671^{* * *}$ & $0,323^{*}$ \\
\hline & 0,157 & 0,164 & 0,166 \\
\hline \multirow[t]{2}{*}{ Edad } & $-0,211$ & $0,422^{*}$ & $-0,306^{*}$ \\
\hline & 0,172 & 0,256 & 0,168 \\
\hline \multirow[t]{2}{*}{$\operatorname{Edad}^{\wedge} 2$} & 0,004 & $-0,010^{*}$ & $0,007^{* *}$ \\
\hline & 0,004 & 0,006 & 0,003 \\
\hline \multirow[t]{2}{*}{$\tau 0$} & $-2,737$ & 2,933 & $-5,432$ \\
\hline & 2,044 & 2,805 & 2,048 \\
\hline \multirow[t]{2}{*}{$\tau 1$} & $-2,219$ & 4,256 & $-4,718$ \\
\hline & 2,036 & 2,814 & 2,047 \\
\hline \multirow[t]{2}{*}{$\tau 2$} & $-1,350$ & 5,361 & $-2,900$ \\
\hline & 2,033 & 2,852 & 2,052 \\
\hline \multirow[t]{2}{*}{$\tau 3$} & $-0,439$ & 5,478 & $-2,560$ \\
\hline & 2,030 & 2,848 & 2,048 \\
\hline Observaciones & 236 & 236 & 236 \\
\hline
\end{tabular}

Errores estándar robustos en paréntesis

${ }^{* * *} \mathrm{p}<0,01,{ }^{* *} \mathrm{p}<0,05,{ }^{\star} \mathrm{p}<0,1$

Fuente: Autor. 
porque no hay una vigilancia adecuada, por ende, no tendrán un castigo por sus comportamientos desviados.

Los resultados también exponen un hecho preocupante y es el desconocimiento de la norma, pues de los estudiantes de la muestra de la Facultad de Administración, el 71,89 \% y el 78,31 \% de la Facultad de Ciencias Humanas desconoce el reglamento que está relacionado con el fraude académico. Mockus (2007) explica que en sociedades en las cuales hay un divorcio entre la ley, la moral y la cultura, se presenta la anomia, es decir, que:

Los fines se logran a cualquier costa, la celebración del éxito y de los resultados desborda de lejos a la celebración del conformarse a las reglas. La sociedad llega a aplaudir y admirar la habilidad para lograr fines buenos mediante acciones contrarias a las reglas (p. 237).

Entonces, las implicaciones de los resultados obtenidos es que cuando el estudiante ni siquiera tiene un conocimiento claro sobre la norma, no podrá existir ese "casamiento" entre la moral, la ley y la cultura, y tendremos una sociedad con síntomas de anomia.

Un punto importante que determinó la tolerancia ante la copia fue la percepción que tienen los estudiantes al considerar este fenómeno como un problema serio. De la muestra obtenida, en la Facultad de Administración el 41,17 \% expresó que no era un problema serio, y el 4,57 \% expresó que no era un problema; el 38,55 \% de la Facultad de Ciencias Humanas expresó que no era un problema serio, y el 6,02 \% expresó que no era un problema. Ariely (2008), después de realizar algunos experimentos sobre la deshonestidad de los estudiantes, afirma que:

Nos preocupa la honestidad, y queremos ser honestos. El problema es que nuestro monitor de honestidad interno solo se activa cuando contemplamos la posibilidad de realizar grandes transgresiones (...) para las pequeñas transgresiones, en cambio... ni siquiera consideramos cómo tales acciones se reflejan en nuestra honestidad (p. 221). 
Aunque la mayoría de los estudiantes expresó que les preocupa la honestidad, se evidencia que, al mismo tiempo, existe una alta frecuencia en incurrir en el fraude. Es decir, la percepción del estudiante que se puede estar entretejiendo es que si incurre en la copia académica, eso no quiere decir que él sea deshonesto, pues cuando se encuentre en una situación mucho más compleja y delicada, obrará de forma correcta. Según lo anterior, se presentan bastantes individuos que hacen pequeñas transgresiones con alta frecuencia. Según Callahan (2004), cuando la copia penetra de tal forma la percepción de los individuos y se tiene la sensación de que "todo el mundo lo hace", un nuevo referente ético surge. Las personas se sienten en desventaja si su comportamiento se ciñe a las normas oficiales, en vez de las que se empiezan a construir. Esto, según Callahan, hace que una cultura del fraude emerja en una sociedad.

Finalmente, algo más profundo debe estar sucediendo en los procesos educativos, pues encontramos esto como un "síntoma" del sistema educativo, mas no como la enfermedad. No es normal que el 77,03\% de los estudiantes encuestados haya sido testigo de ver a otros compañeros incurrir en fraude académico. Ahora, enfocándonos un poco más en la Facultad de Administración, es importante entender que son sus futuros egresados quienes estarán desarrollando algún tipo de actividad económica de alto alcance, y es menester que su proceder se encuentre enmarcado dentro de las normas y leyes de la sociedad, pero también dentro de los principios y valores que la rigen. Dada la alta frecuencia de este fenómeno dentro de los procesos formativos de los estudiantes de pregrado, es importante abrir nuevas discusiones con el fin de plantear posibles alternativas que incidan positivamente en los procesos educativos.

\section{Referencias bibliográficas}

Ariely, D. (2008). Las trampas del deseo: Cómo controlar los impulsos irracionales que nos llevan al error. Madrid, Barcelona: Editorial Ariel S.A. 
Becker, G.S. (1968). Crime and Punishment: An Economic Approach. Journal of Political Economy, 76(2), 169-217.

Bouville, M. (2010). Why is Cheating Wrong? Studies in Philosophy \& Education, 29(1), 67-76.

Callahan, D. (2004). The cheating culture. Why more Americans are doing wrong to go ahead. New York: A Harvest Book/Harcourt Inc.

Chisholm, D. (1992). An Epidemic of Cheating?PS: Political Science and Politics, 25(2), 264-272.

Colnerud, G., \& Rosander, M. (2009). Academic dishonesty, ethical norms and learning. Assessment \& Evaluation in Higher Education, 34(5), 505-517.

Giddens, A. (2009). Sociología (6 $6^{\mathrm{a}}$ ed.) Madrid: Alianza Editorial.

Lipovetsky, G. (2003). Metamorfosis de la cultura liberal ética, medios de comunicación, empresa. Madrid, Barcelona: Anagrama.

Magnus, J., Polterovich, V., Danilov, D. \& Savvateev, A. (Spring, 2002). Tolerance of Cheating: An Analysis across Countries. The Journal of Economic Education, 33(2), 125-135.

Mockus, A. (2007). Pedagogía de la acción política no violenta. En CEPI \& Freddy Cante (Eds.), Poder Social: Algunas posibilidades en Colombia. (pp. 233-260). Bogotá: Editorial Universidad del Rosario.

Morin, E. (1999). Introducción al pensamiento complejo. Barcelona: Editorial Gedisa.

Murdock, T. B., Beauchamp, A. S., and Hinton, A. M. 2008. Predictors of Cheating and Cheating Attributions: Does Classroom Context Influence Cheating and Blame for Cheating? European Journal of Psychology of Education, 23(4), 477-492.

Sims, R. L. (1993). The relationship between academic dishonesty and unethical business practices. Journal of Education for Business, 68, 207-211.

Smyth, L.S., Davis, J.R., \& Kroncke, C.O. (2009). Students' Perceptions of Business Ethics: Using Cheating as a Surrogate for Business Situations. Journal of Education for Business, 84(4), 229-239. 


\section{Anexos}

Figura 1. Promedio de la percepción hacia el estudiante A

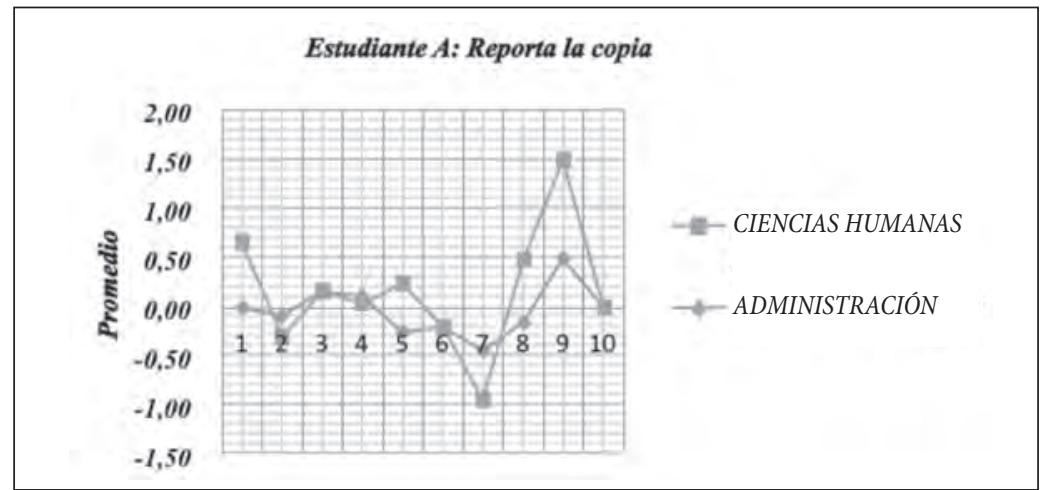

Fuente: Autor.

Figura 2. Promedio de la percepción hacia el estudiante B

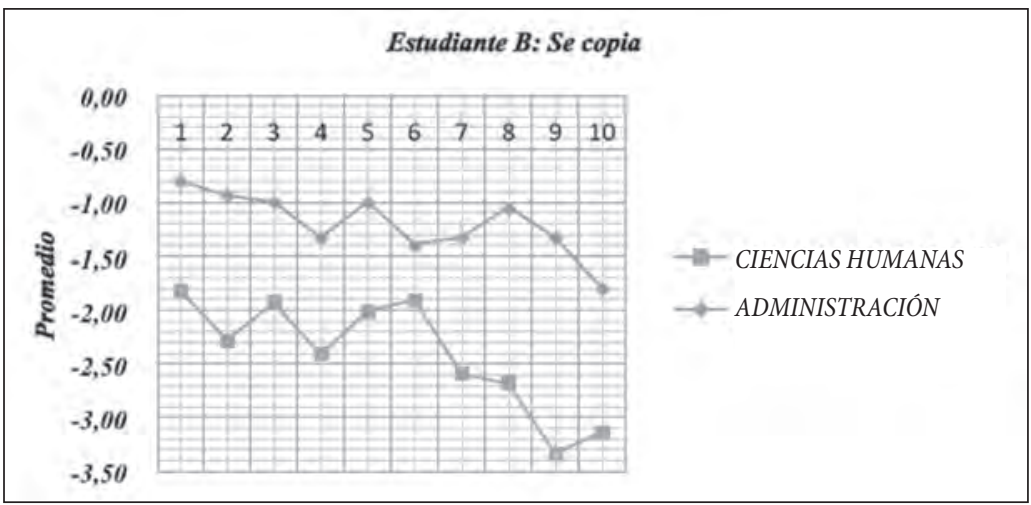

Fuente: Autor. 
Figura 3.Promedio de la percepción hacia el estudiante C

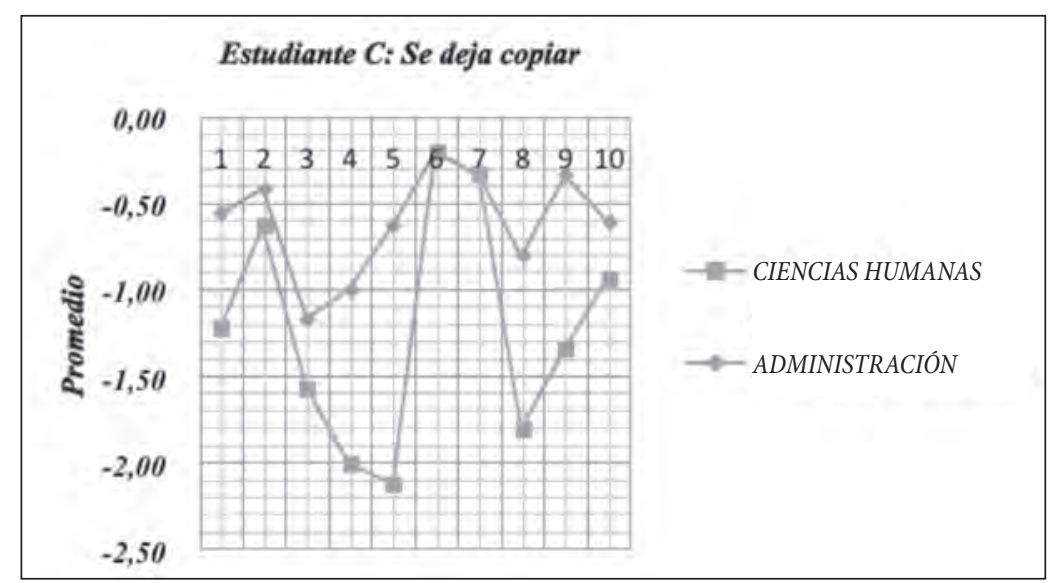

Fuente: Autor. 